\title{
Endoscopic Trans-ethmo-sphenoidal Optic Nerve Decompression for Traumatic Optic Neuropathy: Case Series and Review
}

\author{
${ }^{1}$ Manish Gupta, ${ }^{2}$ Gavinder Singh Bindra, ${ }^{3}$ Ravi Vohra
}

\begin{abstract}
Objective: To assess the visual outcome after endoscopic, endonasal trans-ethmo-sphenoidal optic nerve decompression in patients with traumatic optic neuropathy.

Materials and methods: Prospective study, at tertiary referral center. Study included 12 patients with post-traumatic indirect unilateral optic nerve injury, not responding to medical management with methylprednisolone. These patients underwent optic nerve decompression via trans-ethmo-sphenoidal route using nasal endoscope. Main outcome measure was postoperative visual acuity.
\end{abstract}

Results: Out of the 12 cases of traumatic optic neuropathy (TON) operated upon, nine had good visual outcome.

Conclusion: The endoscopic endonasal trans-sphenoidal approach can decompress the traumatized and swollen optic nerve with excellent visualization of the orbital apex and optic canal, without the morbidity associated with brain retraction as in the conventional transcranial decompression.

Keywords: Blindness acquired, Endonasal trans-sphenoidal approach, Endoscope, Methylprednisolone, Optic canal decompression, Traumatic optic neuropathy.

How to cite this article: Gupta M, Bindra GS, Vohra R. Endoscopic Trans-ethmo-sphenoidal Optic Nerve Decompression for Traumatic Optic Neuropathy: Case Series and Review. Clin Rhinol An Int J 2015;8(3):99-103.

\section{Source of support: Nil}

Conflict of interest: None

\section{INTRODUCTION}

Patients with head injury may present with partial or complete loss of vision due to traumatic optic neuropathy (TON). Direct injuries, caused by penetrating

\footnotetext{
${ }^{1-3}$ Associate Professor

${ }^{1}$ Department of ENT, Gian Sagar Medical College and Hospital Patiala, Punjab, India

${ }^{2}$ Department of Neurosurgery, Gian Sagar Medical College and Hospital, Patiala, Punjab, India

${ }^{3}$ Department of Ophthalmology, Gian Sagar Medical College and Hospital, Patiala, Punjab, India

Corresponding Author: Manish Gupta, Associate Professor Department of ENT, Gian Sagar Medical College and Hospital, Patiala, Punjab, India, Phone: 9915025819, e-mail: manishgupta1217@gmail.com
}

trauma to optic nerve generally results in poor visual recovery, while indirect ones caused by force transmission or fractures following blunt trauma have superior visual outcome. ${ }^{1}$ Of the four segments of optic nerve $(\mathrm{ON})$ intraocular, intraorbital, canalicular and intracranial, the canalicular segment is the most vulnerable to compression, since here it passes through a narrow bony canal and is fixed to periosteum. ${ }^{2}$

Although the international optic nerve trauma study in 1999, found no substantial advantage for either corticosteroid treatment or ON decompression, it had inherent limitations of small sample size. ${ }^{3}$ Moreover, a metaanalysis has evidently demonstrated that medical therapy and/or ON decompression is better than no treatment alone for TON. Comparison of surgical and nonsurgical groups showed that vision improved more significantly in former, especially if the initial visual acuity (VA) was hand movement or more. ${ }^{4}$

Conventional neurosurgical procedure for $\mathrm{ON}$ decompression via transcranial approach causes brain retraction injury. ${ }^{5}$ Endoscopic approach is more effective and less invasive approach for $\mathrm{ON}$ decompression. ${ }^{5}$

\section{MATERIALS AND METHODS}

Thirty patients with head injury presenting to the emergency services with complaints of decreased vision following injury or if unconscious, with loss of pupillary reflex were considered for the study. These were examined by trained ophthalmologist to assess optic functions, such as VA and visual fields (VF). Visual acuity was graded as follows: no light perception, light perception, perception of hand movement, counting fingers and VA on Snellen's chart. Pre- and postoperative VFs were tested by Zeiss Humphrey perimeter. Full field was done in all patients (when possible), while pattern 30-2 or 24-2 was done for ON head function. Fundus provided information on intraocular conditions. Criteria for diagnosis of $\mathrm{ON}$ injury are significant visual loss with an afferent pupillary defect, without significant pathology in anterior or posterior chambers. Non-contrast computed tomogram (NCCT) with axial $2 \mathrm{~mm}$ sections and $1 \mathrm{~mm}$ overlap were performed to evaluate bony structure of optic canal (for 


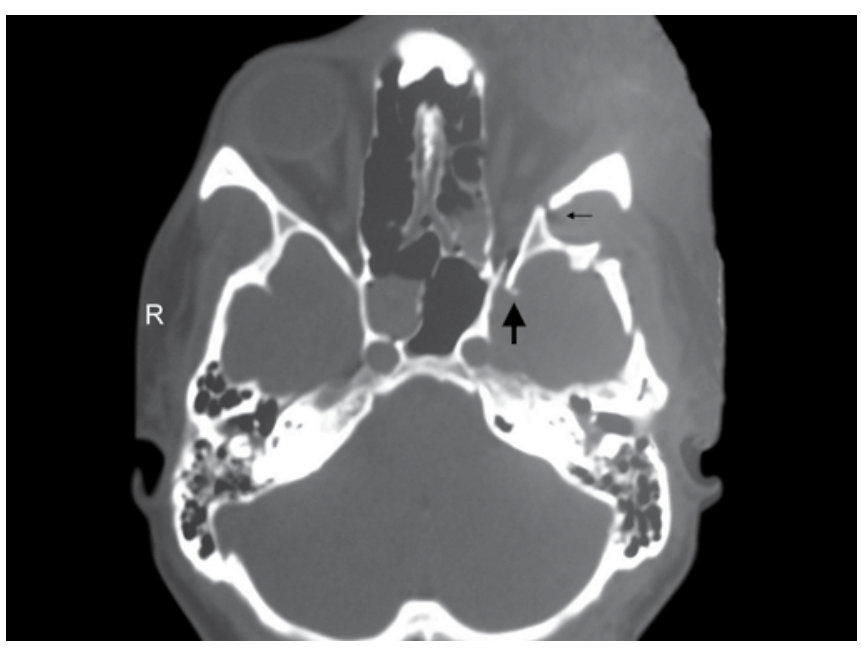

Fig. 1: Axial cut computed tomogram showing fracture of left lesser wing of sphenoid with displacement (thin arrow) and compromise of optic canal (thick arrow)

Flow Chart 1: Management protocol being followed for OND

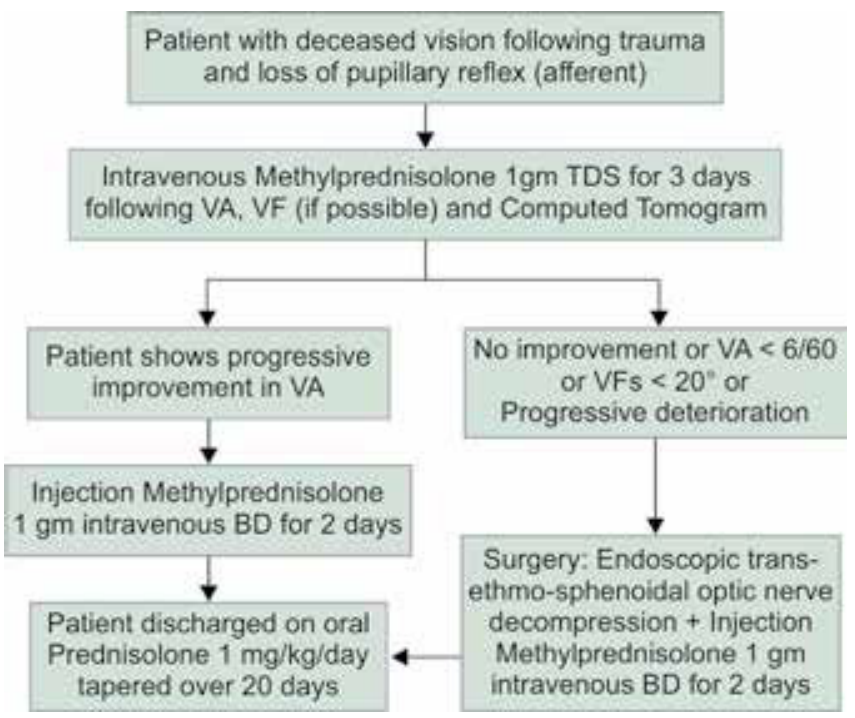

grading) and ethmoid labyrinth (to build road map for surgery) (Figs 1 and 2). The radiology, fundus and VF examination (if possible), pointed to diagnosis of ON injury of canalicular segment.

All patients were initially administered high dose pulsed intravenous steroid therapy using $1 \mathrm{gm}$ of methylprednisolone 8 hourly, for 3 consecutive days and then tapered over time (Flow Chart 1). Visual acuity was monitored daily to assess improvement or worsening of patient's vision in affected eye. Patients not improving with steroid treatment (VA $<6 / 60$ or VFs $<20^{\circ}$ ) or showing progressive deterioration were offered surgical decompression. Eighteen patients showed progressive improvement with steroids, while 12 had no response. These 12 non-responders were taken up for ON decompression after informed consent, presuming preinjury vision in affected eye was equivalent to that of contra-lateral eye.

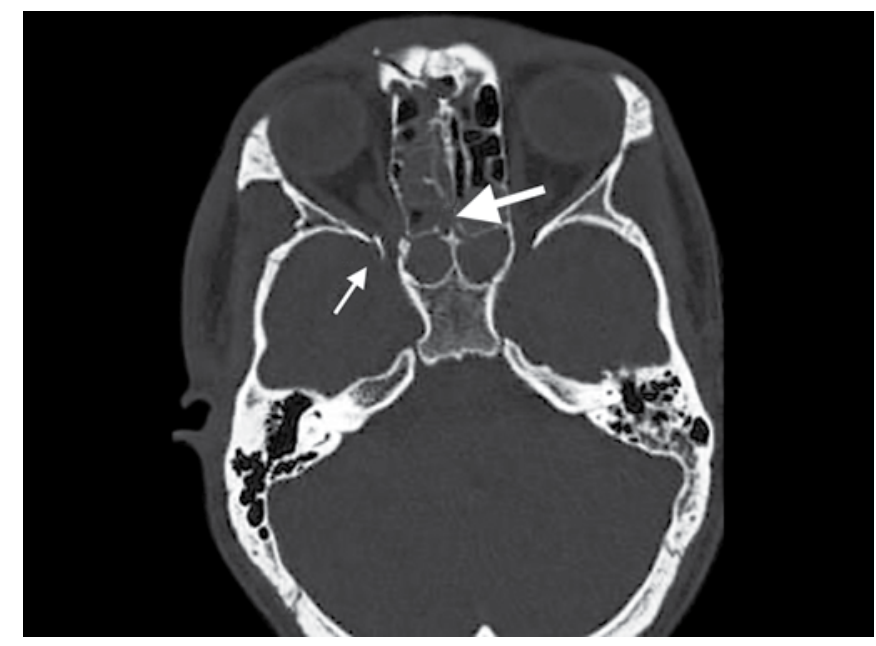

Fig. 2: Axial cut computed tomogram of another patient, showing fracture of right lesser wing of sphenoid compromising optic canal (thin arrow) and fracture of ethmoid labyrinth (thick arrow)

The preoperative VA in these 12 patients ranged from no perception of light (PL-ve) to 6/60. Visual acuity examination was not possible in majority except one, due to worse VA. Severity of injury was graded based on system proposed by Cook et al ${ }^{6}$ (Table 1). Grade of injury is based on fractures of optic canal or posterior orbit and worst recorded vision (mostly immediate post-traumatic vision). In this system of grading, fractures of posterior orbit and optic canal were treated similarly. The interval from onset of visual loss to surgical decompression ranged from 2 to 60 days.

Transnasal endoscopic ipsilateral ethmoidectomy and sphenoidotomy was done under general anesthesia. The optic canal was recognized by bulge on lateral wall of sphenoid, and then followed anteriorly or by following orbital periosteum posteriorly till opening of optic canal. The optic canal was decompressed by removing its bony medial wall in the sphenoid sinus. The posterior limit of bone removal was where nerve curved from an oblique to a transverse direction, nearing chiasmal region (Fig. 3). In all 12 patients nerve sheath was incised using sickle knife in superomedial quadrant of circumference of nerve. The incision was taken, starting from posteriorly toward anteriorly, including anterior fibrous annulus of Zinn and up to $5 \mathrm{~mm}$ of posterior orbital periosteum. After slitting nerve sheath the anesthetist administered Valsalva maneuver, to rule out possible cerebrospinal

Table 1: Grading of traumatic optic nerve injury ${ }^{6}$

\section{Grade 1 No fracture, vision better than 6/60 on Snellen's} chart

Grade 2 No fracture, vision better than PL-ve but less than or equal to $6 / 60$

Grade 3 Fracture present or PL-ve

Grade 4 Fracture present \& PL-ve

PL: Perception of light; - ve $=$ absent 
Endoscopic Trans-ethmo-sphenoidal Optic Nerve Decompression for Traumatic Optic Neuropathy

Table 2: Pre and postoperative vision following transnasal trans-ethmo-sphenoid ON decompression

\begin{tabular}{|c|c|c|c|c|}
\hline $\begin{array}{l}\text { Sl. } \\
\text { no. }\end{array}$ & $\begin{array}{l}\text { Preoperative vision } \\
\text { Lt/ Rt eye }\end{array}$ & $\begin{array}{l}\text { Number of days since injury to } \\
\text { surgery }\end{array}$ & Fracture of optic canal & Postoperative vision \\
\hline 1 & Lt PL-ve & 60 & Yes & No improvement \\
\hline 2 & Lt PL-ve & 5 & Yes & Deceased (brain injury) \\
\hline 3 & Lt HM & 4 & Yes & $6 / 36$ at 1 month \\
\hline 4 & Rt PL-ve & 2 & Yes & $\mathrm{HM}$ at 3 months \\
\hline 5 & Lt FC & 4 & No & $6 / 36$ at 1 month \\
\hline 6 & Lt PL+ve & 5 & No & $6 / 60$ at 1 month \\
\hline 7 & Rt FC & 3 & Yes & $6 / 9$ at 3 months \\
\hline 8 & Rt FC & 7 & No & $6 / 24$ at 1 month \\
\hline 9 & Lt PL+ve & 4 & Yes & $6 / 36$ at 3 months \\
\hline 10 & Lt HM & 5 & Yes & $6 / 36$ at 3 months \\
\hline 11 & Rt HM & 3 & Yes & $6 / 18$ at 1 month \\
\hline 12 & Rt 6/60 & 6 & No & $6 / 24$ at 3 months \\
\hline
\end{tabular}

Rt: right eye; Lt: left eye; PL: perception of light; FC: finger counting; HM: hand movement; -ve: absent; +ve: present

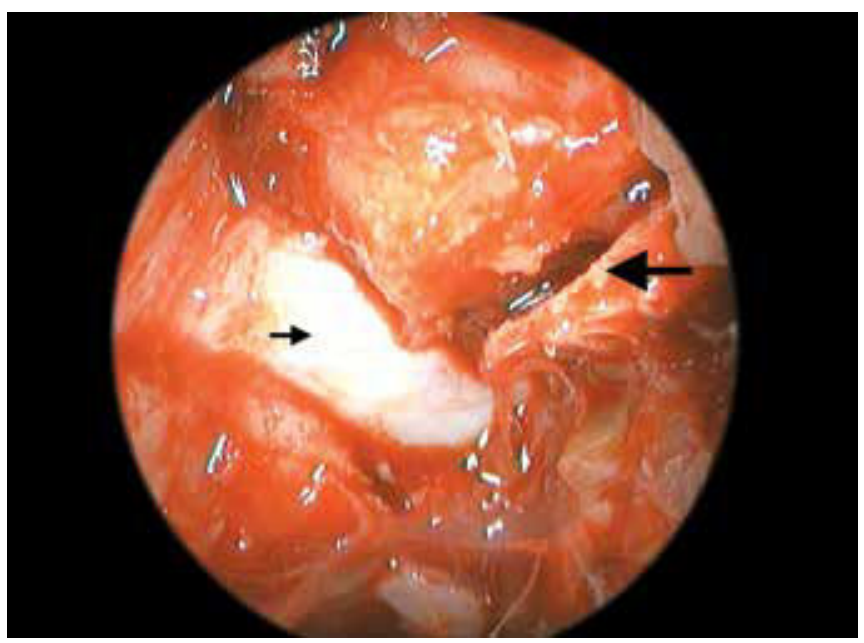

Fig. 3: Intraoperative picture, showing decompressed optic nerve from orbit to chiasma (thin arrow) and a fracture line (thick arrow)

fluid leak. In presence of leak, temporalis fascia graft was placed over opened sheath. Ethmoid gallery and nasal cavity were packed with medicated ribbon gauze for 48 hours. Postoperatively, intravenous antibiotics were instituted for initial 48 hours and subsequently oral antibiotics for next 5 days. Analgesics along with systemic decongestant were prescribed till the pack was in situ. Intravenous methyl-prednisolone $1 \mathrm{gm}$ twice daily was administered for 48 hours postoperatively, followed by oral prednisolone ( $1 \mathrm{mg} / \mathrm{kg}$ body weight/day), which was tapered over 20 days.

Patients were reassessed at 1 week, 1 and 3 months intervals post-surgery for improvements in VA and new complications.

The criteria for improvement in patient's vision was an increase of three lines or more on Snellen's visual chart or change in vision from nonperception to perception of light, from perception of light to recognition of hand movements or from hand motion to finger counting. Treatment was considered successful when vision improved to at least 3/60, based on World Health Organization's (WHO) definition of blindness. ${ }^{7}$

\section{RESULTS}

Thirty patients in study group comprised of 22 males and eight females. The ages ranged from 18 to 70 years, mean age being 32. All 30 patients had sustained the injury in a road traffic accident. Seven had some period of unconsciousness following injury and in three this period was more than 12 hours. Fifteen had unilateral nasal bleed.

Twenty patients had fracture line involving optic canal of which 15 had fracture of ethmoid labyrinth also. The vision of 18 patients improved with steroid therapy alone, and they were discharged on an oral regimen of prednisolone (started with $1 \mathrm{mg} / \mathrm{kg} /$ day for 5 days and subsequently tapered). In 12 patients osseous canal decompression was done via transnasal endoscopic ethmoidectomy and sphenoidotomy. Though two of our patients had intraoperative CSF leak, and was repaired in same sitting, no one had any minor or major complications ranging from synechiae to $\mathrm{ON}$ damage, carotid injury or CSF leak and none had any worsening of VA post-surgery.

Out of the 12 patients that underwent transnasal ON decompression, 10 showed improvement, among these, one had no perception of light while others had partial vision loss preoperatively. One patient deceased due to associated brain injury and one which reported 2 months after injury did not show any improvement (Table 2). The surgical treatment was considered successful (by WHO criteria) $)^{7}$ for nine patients who showed improvement in VA postoperatively.

\section{DISCUSSION}

Optic nerve is prone to compression in canalicular part due to bony surrounding. Compression may occur because of various factors like fracture of bony canal, intraneural contusion (following hemorrhage), secondary vasospasm or veno-occlusion causing edema and 
necrosis. ${ }^{8}$ The visual loss caused by veno-occlusion and compression is usually reversible with early intervention, delayed therapy or progressive compression may lead to arterial obstruction and infarction. It is also likely that midline forehead injury can produce bilateral ON injury while blow over supra-orbital area, temporal area produces unilateral ON injury. ${ }^{9}$

In conscious patients, diagnosis is made with typical history of uni/bilateral visual loss, immediate or delayed type following trauma. In unconscious patients, unilateral fixed, dilated pupil with retained consensual light reflex raises the possibility of unilateral ON injury. This phenomenon is known as 'Marcus Gunn's pupil'. Except a black eye, the cornea, lens, vitreous and fundus reveal no abnormality. Rarely, congestion of disk may be observed. The optic disk atrophy is usually discernable after 4 to 6 weeks of injury. It was present in one of our subjects, who presented 60 days post injury. Visual fields show temporal defect in most cases, due to selective involvement of nasal fibers, as medially orbital bone is thin and more likely to fracture. In the present study, most patients had preoperative vision loss of finger counting, hand movement or poorer, thus VF could not be tested. Only one case had vision of 6/60 with no fracture of optic canal and had VF loss of nasal side.

High resolution computed tomogram is the radiological investigation of choice to demonstrate fracture of optic canal. It may show ethmoid and sphenoid hemorrhage. Magnetic resonance imaging is useful to visualize nerve swelling and contusion. The incidence of optic canal fracture in traumatic blindness has been variously reported from 6 to $92 \% .^{10}$

The relations of $\mathrm{ON}$ to sphenoid and ethmoid sinuses, as classified by DeLano into four types was found to be important in preoperative planning. They are in Type Inerve does not contact or impinge on either sphenoid or posterior ethmoid cells. In Type II-nerve indents sphenoid sinus, without contacting posterior ethmoid cells. In Type III-nerve runs through sphenoid sinus and it is surrounded by pneumatized sinus for at least $50 \%$. In Type IV-nerve courses close to both sphenoid sinus and posterior ethmoid sinus. ${ }^{11}$

Medical treatment with steroids is initiated to reduce swelling or edema of nerve. Mega-dose steroids reduce central nervous system edema, microcirculatory spasm and neural necrosis allowing small nutrient vessels that feed nerve to regain patency. We also used steroids with substantial benefit, thereby obviating the need for surgery in many patients. The group of patients who showed no improvement or with minimal improvement and then remain static were subjected to surgery. Surgical decompression is effective because it releases compartmental syndrome and improves blood flow to anterior $\mathrm{ON}_{1}^{12}$ giving a chance of recovery to partially damaged ON. A study has revealed that demyelinated optic nerves remyelinate spontaneously, albeit partially, indicating that mature mammalian $\mathrm{ON}$ does have the ability to repair itself if axonal structure is maintained. ${ }^{13}$

Various surgical approaches, such as transfrontal craniotomy, extranasal transethmoidal, transantral ethmoidal, lateral facial and endoscopic procedures have been developed to access ON. ${ }^{14}$

The endoscopic technique is advocated for a variety of surgeries of pituitary and anterior skull base tumors. 5,15 It provides a magnified and enhanced illuminated operative view than conventional cranial approaches, giving superior delineation of anatomical relations. Endoscopic technique is safe, effective and less invasive with early recovery and is less demanding for patients in postoperative period. Furthermore, it avoids external disfigurement and preserves olfaction.

The otolaryngologists prefer endonasal approach for ON decompression using trans-ethmoid trans-sphenoid route, going more laterally. This approach provides shortest straight route to optic canal. While some surgeons have a preference for direct sphenoidotomy through natural ostium, it is disadvantageous because of long distance to optic canal.

The slit opening of $\mathrm{ON}$ sheath remains controversial. ${ }^{16}$ Some authors advocate decompression of nerve sheath arguing that post-traumatic compartmental syndrome is best relieved by releasing constricting sheath enveloping the nerve. ${ }^{17}$ While some surgeons shun nerve sheath slitting, doubting its efficacy and potential risk of cerebrospinal fluid leak, threat of disrupting nerve fascicles or a possible hazard to pia vessels and ophthalmic artery. ${ }^{18}$ In a study on 57 patients $\mathrm{ON}$ sheath incision has been found to confer improved result than just osseous decompression, especially in subjects without optic canal fracture. ${ }^{19}$ The patients with external compression of $\mathrm{ON}$ by displaced fracture or extraneural hematoma respond well to bony decompression alone, while patients with internal compression due to edema or hematoma would be better relieved by a further slitting of ON sheath and annulus of Zinn. The layers of $\mathrm{ON}$ sheath as viewed from a medial trans-sphenoidal approach include periosteal and dural layers of dura mater, arachnoid sheath, subarachnoid space and pial sheath, which is adherent to nerve. Placing fascial graft over the incision site, as in the present study, circumvents the probability of CSF leaks later. It has also been suggested to apply fibrin glue during repair. ${ }^{17}$ The ophthalmic artery traverses in $\mathrm{ON}$ sheath lateral to nerve and is not encountered in medial trans-sphenoid approach. However, occasionally it may 
be inferior or inferomedial to nerve, thus it is a usual practice to incise nerve sheath in superomedial quadrant of nerve circumference. ${ }^{17}$

The timing of surgery is also important, because in one study visual improvement was achieved in $70 \%$ when treatment was initiated within 7 days of injury, whereas only $24 \%$ improved if treatment was delayed. ${ }^{14}$ Similar observations have been made in our study, though the study group was small. Time lapse after injury, degree of visual loss and computed tomographic evidence of canalicular fractures were found to be significant prognostic factors.

Surgical complications include CSF leakage, direct injury to $\mathrm{ON}$ and injury to internal carotid, ethmoid or ophthalmic artery. Thus, it is logical to give initial treatment with high dose of steroids, with surgical decompression being reserved for only for patients who either do not improve or in whom vision progressively deteriorates. However, no such complications were observed in our patients.

\section{REFERENCES}

1. Warner JEA, Lessell S. Traumatic optic neuropathy. Int Ophthalmol Clin 1995;35:57-62.

2. Steinsapir KD, Goldberg RA. Traumatic optic neuropathies. In: Walsh and Hoyt's Clinical Neuro-Ophthalmology. 5th ed. Baltimore, Md: William \& Wilkins; 1997. p. 715-739.

3. Levin LA, Beck RW, Joseph MP, Seiff S, Kraker R. The treatment of traumatic optic neuropathy: the international optic nerve trauma study. Ophthalmol 1999;106:1268-1277.

4. Mine S, Yamakami I, Yamaura A, Hanawa K, Ikejiri M, Mizota A, et al. Outcome of traumatic optic neuropathy. Comparison between surgical and nonsurgical treatment. Acta Neurochir (Wien) 1999;141:27-30.

5. Cappabianca P, Cavallo LM, Divitiis ED. Endoscopic endonasal transsphenoidal surgery. Neurosurg 2004;55:933-941.
6. Cook MW, Levin LA, Joseph MP, Pinczower EF. Traumatic optic neuropathy. A meta-analysis. Arch Otolaryngol Head Neck Surg 1996;122:389-392.

7. Data on blindness throughout the world (editorial). WHO Chron 1979;33:275-283.

8. Steinsapir KD, Goldberg RA. Traumatic optic neuropathy. Surv Ophthalmol 1994;38:487-518.

9. Mahapatra AK. Bilateral optic nerve injury. Neurol India 1999;47:92-93.

10. Osguthorpe JD, Sofferman RA. Optic nerve decompression. Otolaryngol Clin North Am 1988;21:155-169.

11. DeLano MC, Fun FY, Zinreich SJ. Relationship of the optic nerve to the posterior paranasal sinuses: a CT anatomic study. Am J Neuroradiol 1996;17:669-675.

12. Sergott RC. Optic nerve sheath decompression: neuropathologic, clinical and hemodynamic results and rationale. Tr Am Ophth Soc 1991;89:675-720.

13. Rajniganth MG, Gupta AK, Gupta A, Bapuraj JR. Traumatic optic neuropathy: visual outcome following combined therapy protocol. Arch Otolaryngol Head Neck Surg 2003; 129:1203-1206.

14. Acheson JF. Optic nerve disorders: role of canal and nerve sheath decompression surgery. Eye 2004;18:1169-1174.

15. Dehdashti AR, Ganna A, Karabatsou K, Gentili F. Pure endoscopic endonasal approach for pituitary adenomas: Early surgical results in 200 patients and comparison with previous microsurgical series. Neurosurg 2008;62: 1006-1017.

16. Luxenberger W, Stammberger H, Jebeles JA, Walch C. Endoscopic optic nerve decompression: the Graz experience. Laryngoscope 1998;108:873-882.

17. Onofrey CB, Tse DT, Johnson TE, Neff AG, Dubovy S, Buck BE, et al. Optic canal decompression: a cadaveric study of the effects of surgery. Ophthal Plast Reconstr Surg 2007;23: 261-266.

18. Thaker A, Tandon DA, Mahapatra AK. Surgery for optic nerve injury: should nerve sheath incision supplement osseous decompression? Skull Base 2009;19:263-272.

19. Sergott RC, Brown MJ, Silberberg DH. Remyelination follows antibody-induced central nervous system demyelination. Ann Neurol 1986;20:94-98. 